\title{
Exposure as Collected Sponsor Defined Identifier
}

National Cancer Institute

\section{Source}

National Cancer Institute. Exposure as Collected Sponsor Defined Identifier. NCI

Thesaurus. Code C117470.

One or more sponsor defined characters used to identify, name, or characterize the collected exposure. 